03;04

\title{
Создание гиперзвуковых течений мощным импульсным капиллярным разрядом
}

\author{
(C) А.С. Пащина, Р.Е. Кармацкий, А.И. Климов
}

Объединенный институт высоких температур РАН, Москва

E-mail: fgrach@mail.ru

Поступило в Редакцию 9 марта 2017 г.

Экспериментально показана возможность использования мощного импульсного капиллярного разряда для получения квазистационарных высокоскоростных потоков плазмы с характерными значениями чисел Маха $M=3-10$ и температур $T=3000-6000 \mathrm{~K}$. В атмосфере разреженного газа $\left(p_{\infty}<10\right.$ Torr $)$ поперечный размер потока превышает $d>3 \mathrm{~cm}$, а продолжительность рабочего цикла может быть доведена до сотен миллисекунд,что представляет интерес в задачах лабораторного моделирования физико-химических и газодинамических эффектов взаимодействия тел с гиперзвуковыми течениями. Обнаружены сильная температурная неравновесность (отношение колебательной и вращательной температур достигает $T_{v} / T_{r}=3$ и более) и аномально низкие значения эффективного показателя адиабаты, что свидетельствует об интенсивной наработке многоатомных молекул и конденсированных частиц в углеродсодержащей плазме.

DOI: $10.21883 /$ PJTF.2017.22.45264.16767

Высокоскоростные плазменные струи находят широкое применение в научных исслдованиях и технических приложениях: физике плазмы, спектроскопии, газодинамике, космической технике, плазменных технологиях и др. Одним из актуальных направлений, предполагающих использование плазменных струй, является динамика гиперзвукового полета, а также родственные проблемы. Сложность и дороговизна натурных экспериментов [1], а также исследований на созданных для этой цели крупномасштабных стендах (см., например, [2]) приводят к необходимости поиска менее затратных способов моделирования гиперзвукового полета в лабораторных условиях, позволяющих оперативно решать отдельно взятые задачи с менее жесткими требованиями к соблюдению критериев подобия и параметров потока. В настоящее время для этой цели применяются различные методы, основанные на 
использовании индукционного высокочастотного разряда [3], катодной дуги низкого давления [4], рельсотронного ускорителя [5,6], магнитноплазменного компрессора [7]. Каждому из этих методов присуща своя специфика, которая определяет их область применения в соответствии с возможностью получения требуемых параметров потока (скорости, температуры, концентарции заряженных частиц, длительности рабочего цикла) в условиях конкретного эксперимента (состав, давление окружающей атмосферы и др.).

Еще одним методом получения высокоскоростных потоков плазмы является использование мощного импульсного разряда в капилляре c аблирующей стенкой. Рабочим телом в таком разряде является вещество стенки капилляра, которое при протекании тока разряда испаряется под воздействием тепловых потоков и поступает в канал разряда, где ионизуется и далее выбрасывается из капилляра в виде плазменной струи. Свойства плазменных струй, инжектируемых в воздух при атмосферном давлении, достаточно детально изучались рядом исследовательских групп (обзор работ представлен в [8]). В то же время детальных исследований свойств таких струй при их инжекции в разреженную атмосфру не проводилось. Согласно результатам предварительных экспериментов истечение плазменной струи в разреженную атмосферу сопровождается ее расширением, понижением газовой температуры и концентрации заряженных частиц. При давлениях менее $p_{\infty}<10$ Torr поперечный размер струи увеличивается до $20-30 \mathrm{~mm}$, что сравнимо с характерными размерами потоков, создаваемыми упомянутыми выше способами, и позволяет рассмотреть возможность использования капиллярного разряда в лабораторных экспериментах по моделированию условий гиперзвукового полета. Обнадеживающими предпосылками для этого служит высокая скорость потока плазмы (до $10-25 \mathrm{~km} / \mathrm{s}$ ), ожидаемое снижение концентрации заряженных частиц до требуемых значений $n_{e}=10^{12}-10^{13} \mathrm{~cm}^{-3}$, принципиально неограниченный диапазон давлений окружающего газа, продолжительное время рабочего цикла (единицы-сотни миллисекунд), более чем на три порядка превышающее времена работы рельсотронных ускорителей и магнитоплазменного компрессора.

Цель настоящей работы - оценка возможности использования плазменной струи, инжектируемой в разреженную атмосферу, в качестве источника высокоскоростного (гиперзвукового) потока, а также оценка газодинамических параметров такого потока.

Письма в ЖТФ, 2017, том 43, вып. 22 
Для получения плазменного потока используется капиллярный разрядник, детальное описание которого приведено в [8]. Состав инжектируемой плазмы определяется материалом стенки капилляра, в качестве которого используется полиметилметакрилат (химическая формула $\left.\mathrm{C}_{5} \mathrm{H}_{8} \mathrm{O}_{2}\right)$. Начальный диаметр капилляра составляет $1 \mathrm{~mm}$, глубина $5 \mathrm{~mm}$. В качестве источника питания разряда используется емкостный накопитель, обеспечивающий следующие параметры разрядного импульса: мощность разрядного импульса $N=15-40 \mathrm{~kW}$, форма тока разряда - полуволна синусоиды, амплитуда разрядного тока $I_{m}=90-160 \mathrm{~A}$, длительность разряда $T_{1 / 2}=7.5 \mathrm{~ms}$. Разрядник размещался в вакуумной камере, минимальная величина остаточного давления в которой составляет $p_{\infty}=3$ Torr. Вдоль оси капилляра на заданном расстоянии от его среза $(1.5-5 \mathrm{~cm})$ устанавливались пробные тела различной формы: сфера, конус, несимметричный клин и др. Эксперименты проводились в атмосфере воздуха в диапазоне давлений $p=3-300$ Torr.

Изучение картины течения проводилось путем регистрации собственных изображений выбранного участка газоплазменного потока с помощью высокоскоростной видеокамеры MotionPro N3 (длительность экспозиции $1 \mu \mathrm{s}$, частота кадров $4-5 \mathrm{kHz}$ ). С помощью спектрометра AvaSpec UL-2048 производилась регистрация спектров излучения плазмы в диапазоне длин волн $280-800 \mathrm{~nm}$, на основе которых также получены данные о компонентном составе и выполнены оценки основных параметров плазмы: концентрации заряженных частиц, колебательной и вращательной температур.

Уже первые пуски показали, что течение в окрестности пробных тел соответствует типичной картине обтекания сверхзвуковым потоком (рис. 1). Перед телом образуется ударная волна, форма и пространственное положение которой определяются геометрией обтекаемого тела и параметрами набегающего потока. Интенсивность излучения плазмы в окрестности ударной волны резко изменяется: перед ударной волной наблюдается зона с пониженной интенсивностью излучения, в то время как в зоне торможения (за ударной волной) интенсивность излучения существенно возрастает. Именно граница резкого изменения интенсивности излучения использовалась в наших экспериментах для определения пространственного положения ударной волны. Определенную уверенность в правомерности применения такого подхода дают результаты исследований других авторов, проводивших эксперименты

Письма в ЖТФ, 2017, том 43, вып. 22 

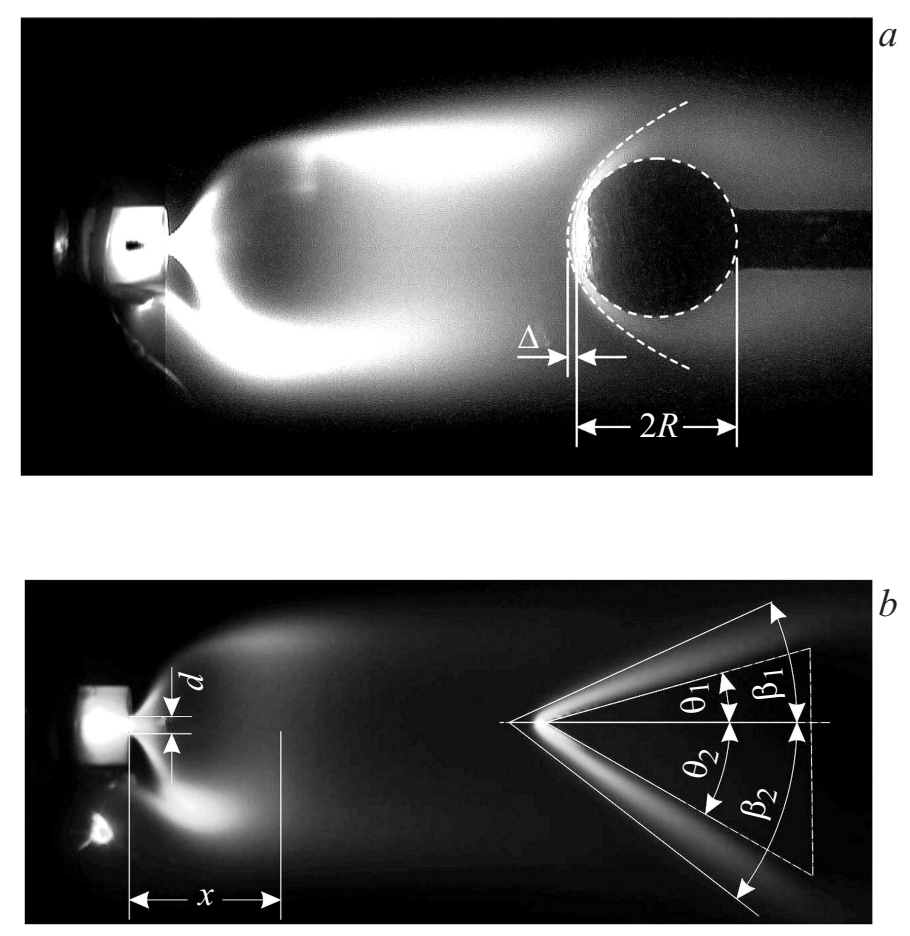

Рис. 1. Типичная картина сверхзвукового обтекания сферы $(a)$ и несимметричного клина $(b)$. Буферный газ-воздух, давление в камере $p_{\infty}=3$ Torr.

при схожих условиях [9], в том числе с применением модифицированных теневых и интерференционных методов визуализации потока [10]. Форма и пространственное положение ударной волны изменяются в течение разрядного импульса в соответствии с изменением скорости потока плазмы, зависящей от текущего значения мощности разряда.

Оценка газодинамических параметров потока производилась двумя независимыми способами: 1) на основе анализа ударно-волновой структуры начального участка струи; 2) на основе анализа пространственного положения отошедшей ударной волны. В первом способе использована зависимость положения центрального скачка уплотнения относительно 
среза капилляра $x_{C}$ от числа Маха [11]

$$
\frac{x_{C}}{d}=M^{\frac{1}{\gamma-1}}\left[\left(\frac{\gamma-1}{2}\right)^{\frac{\gamma}{\gamma-1}} \frac{\gamma+1}{4.8 \gamma}\right]^{1 / 2},
$$

где $d$ - диаметр капилляра, $\gamma$ - показатель адиабаты.

Во втором способе использована связь геометрических параметров отошедшей ударной волны и числа Маха набегающего потока. Для клина эта связь устанавливается известной аналитической зависимостью

$$
\frac{1}{M^{2}}=\frac{\gamma+1}{2} \sin \beta \cos \beta \operatorname{tg}(\beta-\theta)-\frac{\gamma-1}{2} \sin ^{2} \beta,
$$

а для сферы может быть представлена эмпирической зависимостью [12]

$$
\frac{\Delta}{R}=0.76 \frac{\gamma-1}{\gamma+1}+\frac{0.23}{(M-1)^{1.43}} .
$$

Здесь $\beta, \theta-$ угол отошедшей ударной волны и угол клина относительно направления набегающего потока соответственно, $\Delta, R-$ отход ударной волны и радиус сферы соответственно.

Оба метода в границах их применимости приводят к близким результатам оценки числа Маха, величина которого зависит от энергии разрядного импульса, размеров капилляра и остаточного давления в камере. Зарегистрированный в проведенной серии экспериментов диапазон изменения чисел Маха составляет $M=3-10$.

Точность оценок числа Маха существенно зависит от значений эффективного показателя адиабаты, который входит в зависимости $(1),(2)$. Значение этого параметра для многокомпонентной плазмы капиллярного разряда заранее неизвестно, что приводит к необходимости одновременного определения обоих параметров ( $M$ и $\gamma)$ по результатам одного пуска. Такая возможность существует для несимметричных моделей, в частности несимметричного клина (рис. $1, b$ ). Пример зависимости числа Маха от эффективного показателя адиабаты, построенной на основе зависимости (1) по результатам одного из пусков, представлен на рис. 2. На этом рисунке сплошные линии $M\left(\theta_{1}, \beta_{1}\right)$, и $M\left(\theta_{2}, \beta_{2}\right)$ построены для средних значений углов $\beta_{1}$ и $\beta_{2}$ ударной волны, отошедшей соответственно от верхней и нижней поверхности клина (рис. $1, b$ ), а штриховыми линиями обозначен диапазон отклонения этих

Письма в ЖТФ, 2017, том 43, вып. 22 


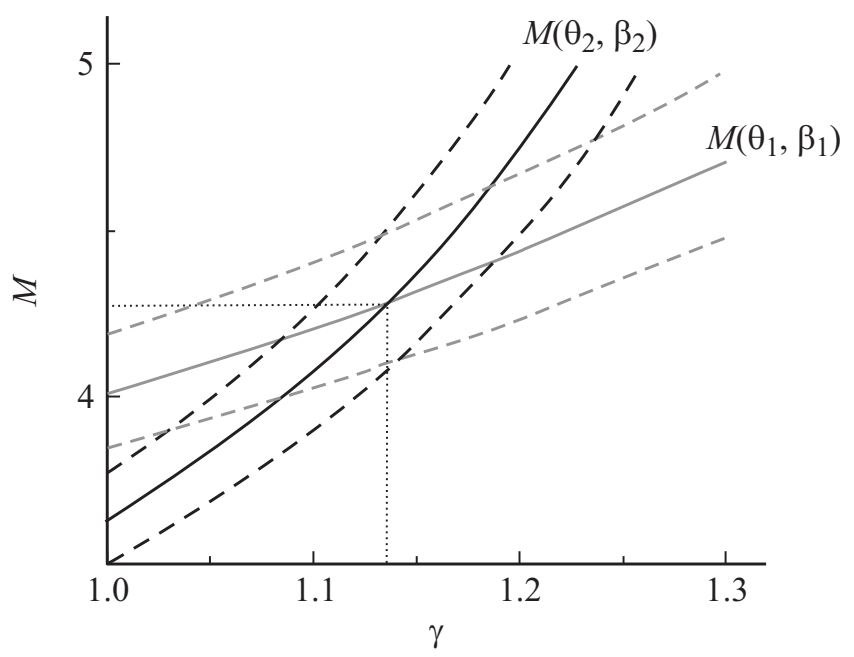

Рис. 2. Пример зависимости числа Маха от эффективного показателя адиабаты, полученной по результатам обтекания несимметричного клина: $\theta_{1}=15.6^{0}$, $\beta_{1}=24.9 \pm 0.60^{\circ}, \theta_{2}=29.9^{\circ}, \beta_{2}=38.7 \pm 0.6^{\circ}$, буферный газ - воздух, давление в камере $p_{\infty}=3$ Torr. Углы $\theta$ и $\beta$ соответствуют обозначениям на рис $1, b$.

зависимостей от их среднего значения, обусловленный погрешностью определения углов $\beta_{1}$ и $\beta_{2}\left( \pm 0.6^{\circ}\right)$. Точка пересечения кривых $M\left(\theta_{1}, \beta_{1}\right)$ и $M\left(\theta_{2}, \beta_{2}\right)$ дает значения $M$ и $\gamma$. Среднее значение эффективного показателя адиабаты для данного примера составляет $\gamma=1.13 \pm 0.1$. Близкий результат получается путем сравнительного анализа экспериментальных данных по обтеканию сферы (рис. 1,a) и эмпирической зависимости (2), верхнее значение эффективного показателя адиабаты в этом случае не превышает $\gamma \leqslant 1.12$.

Низкие значения эффективного показателя адиабаты служат признаком значительной концентрации многоатомных молекул и конденсированных частиц в газоплазменном потоке. Источником многоатомных молекул и конденсированных частиц является входящий в состав материала стенки капилляра углерод. Оптимальные условия роста конденсированных частиц достигаются в ограниченном диапазоне температур $T=1500-4500 \mathrm{~K}$ [13], что, как показано в [14], является причиной немонотонной зависимости эффективного показателя адиабаты 
углеродсодержащей плазмы от температуры. При инжекции плазменной струи в разреженную атмосферу такие условия могут выполняться в большей части ее объема, что подтверждается результатами оценки температуры по зарегистрированным спектрам излучения плазмы. Диапазоны значений колебательной и вращательной температур, определенные по молекулярным полосам Свана радикала $\mathrm{C}_{2}$, составляют $T_{V}=9000-14000 \mathrm{~K}$ и $T_{r}=3800-6000 \mathrm{~K}$ соответственно. Обращает на себя внимание большой отрыв (до 3 и более раз) колебательной и вращательной температур. Сильная температурная неравновесность, очевидно, является результатом избирательного влияния газодинамики течения на параметры плазменных компонентов (электронов и тяжелых частиц) вследствие существенного различия их масс.

Таким образом, результаты первых экспериментов свидетельствуют о возможности использования импульсного капиллярного разряда в качестве простого и эффективного способа получения в разреженной атмосфере квазистационарных потоков плазмы в диапазоне параметров, представляющих интерес для лабораторного моделирования физикохимических и газодинамических эффектов и экспериментального изучения процессов взаимодействия тел с высокоскоростными газоплазменными потоками. Обнаруженные особенности эрозионной плазмы, такие как сильная температурная неравновесность и низкие значения эффективного показателя адиабаты, позволяют по-новому подойти к решению многих фундаментальных задач магнитопламенной аэродинамики и, вполне вероятно, могут представлять интерес в других областях науки и техники. В дальнейшем представляется целесообразным детальное изучение теплофизических электрофизических и газодинамических свойств таких потоков с целью определения режимов разряда, удовлетворяющих требованиям к проведению модельных экспериментов. Важным шагом в дальнейшей работе должно стать повышение пространственного разрешения картин обтекания за счет применения модифицированных теневых методов [10], обеспечивающих возможность непосредственного контроля скачков уплотнения в условиях низких градиентов плотности.

\section{Список литературы}

[1] Hayes D.T., Rotman W. // AIAA J. 1973. V. 11. N 5. P. 675-682.

[2] Poniaev S.A., Kurakin Y.A, Schmidt A.A, Bobashev S.V., Steffens L., Esser B., Gulhan A. // J. Phys. Conf. Ser. 2014. V. 572. P. 1-5.

6 Письма в ЖТФ, 2017, том 43, вып. 22 
[3] Lemmer K.M., Gallimore A.D., Smith T.B. // Plasma Sources Sci. Technol. 2009. V. 18. N 2. P. 25019.

[4] Shashurin A., Zhuang T., Teel G., Keidar M., Kundrapu M., Loverich J., Beilis I., Raitses Y. // J. Spacecr. Rockets. 2014. V. 51. N 3. P. 838-846.

[5] Бобашев С.В., Жуков Б.Г., Куракин Р.О., Поняев С.А. // Письма в ЖТФ. 2016. T. 42. B. 6. C. 62-69.

[6] Бобашев С.В., Резников Б.И., Жуков Б.Г., Куракин Р.О., Поняев С.А. // Письма в ЖТФ. 2015. Т. 41. В. 13. С. 64-71.

[7] Mashek I.C., Lashkov V.A., Anisimov Y.I., Kolesnichenko Y.F. // 44th AIAA Aerosp. Meet. Exhib. Reno. NV, 2006. Paper 2006-1458.

[8] Пащина А.С., Ефимов А.В., Чиннов В.Ф. // ТВТ. 2016. Т. 54. № 4. С. 513528.

[9] Zander F., Gollan R.J., Jacobs P.A., Morgan R.G. // Shock Waves. 2014. V. 24. N 2. P. $171-178$.

[10] Бойко В.М., Оришич А.М., Павлов А.А., Пикалов В.В. Методы оптической диагностики в аэрофизическом эксперименте / Под ред. В.М. Фомина. Новосибирск: НГУ, 2009. 450 с.

[11] Crist S., Sherman P.M., Glass D.R. // AIAA J. 1966. V. 4. № 1. P. 68-71.

[12] Масленников В.Г. // Аэрофизические исследования сверхзвуковых течений / Под ред. Ю.А. Дунаева, М.-Л.: Наука, 1967. С. 256-264.

[13] Eremin A.V. // Prog. Energy Combust. Sci. 2012. V. 38. N 1. P. 1-40.

[14] Резников Б.И., Алексеев Н.И., Бобамев С.В., Жуков Б.Г., Поняев С.А., Куракин Р.О., Розов С.И. // ЖТФ. 2011. Т. 81. В. 8. С. 48-52. 